\title{
Population processes sampled at random times
}

\author{
Luisa Beghin* $\quad$ Enzo Orsingher ${ }^{\dagger}$
}

\begin{abstract}
In this paper we study the iterated birth process of which we examine the first-passage time distributions and the hitting probabilities. Furthermore, linear birth processes, linear and sublinear death processes at Poisson times are investigated. In particular, we study the hitting times in all cases and examine their long-range behavior.

The time-changed population models considered here display upward (birth process) and downward jumps (death processes) of arbitrary size and, for this reason, can be adopted as adequate models in ecology, epidemics and finance situations, under stress conditions.

Keywords and phrases: Yule-Furry process, linear and sublinear death processes, hitting times, extinction probabilities, first-passage times, Stirling numbers, Bell polynomials. AMS Mathematical Subject Classification (2010): 60G55, $60 J 80$.
\end{abstract}

\section{Introduction}

We study here different compositions of point processes, say $K(t):=L(H(t)), t \geq 0$, where $L$ and $H$ are mutually independent and can possibly be birth, death or homogeneous Poisson processes. These compositions arise in the analysis of different population models, when, in some specific experimental circumstances, the time $t$ must be replaced by a stochastic process $H(t), t \geq 0$. In our analysis we concentrate our attention on the case where the time increments are unit-valued and thus are properly represented by point processes, such as linear birth and Poisson processes. In these cases the composed process can be regarded as a randomly sampled population model.

The simplest case of the composition of two independent homogeneous Poisson processes (with different rates) has been already studied in [11. More recently, the iterated Poisson process has been considered in [6.

Our investigation concentrates here on the distribution of the first-passage times, i.e.

$$
T_{k}:=\inf \{t \geq 0: K(t)=k\}, \quad k \geq 0,
$$

which, in this context, substantially differ from the upcrossing and downcrossing times, i.e.

$$
U_{k}:=\inf \{t \geq 0: K(t) \geq k\}, \quad V_{k}:=\inf \{t \geq 0: K(t) \leq k\}, \quad k \geq 0 .
$$

Indeed, the composed processes $K$ jump over (resp. under) any level $k$ with positive probability. The hitting probabilities $\operatorname{Pr}\left(T_{k}<\infty\right)$ display different behaviors. For example, for the iterated birth process $K(t)=B_{1}\left(B_{2}(t)\right)$ (with $B_{1}, B_{2}$ independent linear birth processes), the probabilities $\operatorname{Pr}\left(T_{k}<\infty\right)$ attain their maximal value at $k=3$. In the linear death process at Poisson distributed time the hitting probabilities display an oscillating behavior, which can be perceived when the initial size of the population is sufficiently large.

Furthermore, we observe that in all the cases considered here the hitting probabilities $\operatorname{Pr}\left(T_{k}<\infty\right)$ depend only on the parameter of the outer process.

*e-mail: luisa.beghin@uniroma1.it

†e-mail: enzo.orsingher@uniroma1.it 
Moreover, the processes $K$ display sample paths with upward or downward jumps of arbitrary size. Other models with the same feature, such as the space-fractional Poisson processes or, in general, time-changed Poisson processes with Bernštein subordinators, have been analyzed in [13. Multiple jumps are also displayed by the generalized fractional birth processes studied in [1]. As in this last work, this property is reflected in the form of the equation governing the state probabilities $p_{k}(t):=\operatorname{Pr}\{K(t)=k\}, k \geq 0$, where the time derivative $p_{k}^{\prime}(t)$ is shown to depend, also here, on all $p_{k-j}(t)$, for $j=0, \ldots, k$.

In particular, our analysis concerns the following specific cases:

- $B_{1}\left(B_{2}(t)\right), t \geq 0 \quad($ section 3$)$

- $B(N(t)), t \geq 0 \quad($ section 4$)$

- $D(N(t)), t \geq 0 \quad($ section 5$)$

- $\widetilde{D}(N(t)), t \geq 0 \quad($ section 6$)$,

where $B_{1}$ and $B_{2}$ are independent linear birth (Yule-Furry) processes, $N$ is the homogeneous Poisson process, $D$ is the linear death process and $\widetilde{D}$ is the sublinear death process. The latter is characterized by the fact that the probability of a further death in $[t, t+d t)$ depends on the number of deaths recorded up to time $t$ (unlike the linear case where this probability depends on the number of surviving individuals). The sublinear birth process was introduced in 9, while the sublinear death process has been investigated in [12, in a fractional context.

The processes considered here can be useful to model the population evolution sampled at Poisson times. The iterated birth process can be more appropriate for the cases where the time separating occurrences is rapidly decreasing. This structure can be applied, for example, in some experimental studies of diseases or epidemic diffusions.

Time-changed birth processes of different forms have been studied in [7] and [8, with various applications to finance. Time-changed Poisson and birth processes arise also in the study of fractional point processes (see, for example, [10], 3], 4] and [5]).

\section{Notations}

\section{List of the main symbols}

$\begin{array}{lll} & \text { PROCESS } & \text { STATE PROBAB. } \\ \text { Linear birth } & B_{\alpha}(t) & \\ \text { Poisson } & N_{\lambda}(t) & \\ \text { Linear death } & D_{\mu}(t) & p_{k}^{D}(t), 0 \leq k \leq n_{0} \\ \text { Sublinear death } & \widetilde{D}_{\mu}(t) & p_{k}^{\widetilde{D}}(t), 0 \leq k \leq n_{0} \\ \text { Iterated birth } & \mathcal{Z}(t)=B_{\alpha}\left(B_{\lambda}(t)\right) & q_{k}^{\mathcal{Z}}(t), k \geq 1 \\ \text { Linear birth at Poisson times } & \mathcal{X}(t)=B_{\alpha}\left(N_{\lambda}(t)\right) & q_{k}^{\mathcal{X}}(t), k \geq 1 \\ \text { Linear death at Poisson times } & \mathcal{Y}(t)=D_{\mu}\left(N_{\lambda}(t)\right) & q_{k}^{\mathcal{Y}}(t), 0 \leq k \leq n_{0} \\ \text { Sublinear death at Poisson times } & \widetilde{\mathcal{Y}}(t)=\widetilde{D}_{\mu}\left(N_{\lambda}(t)\right) & q_{k}^{\widetilde{Y}}(t), 0 \leq k \leq n_{0}\end{array}$

where $n_{0} \geq 1$ is the number of the initial components of the population and $\lambda, \mu, \alpha>0$.

\section{The iterated linear birth process}

Let us first consider the composition of a linear birth (Yule-Furry) process $B_{\alpha}:=B_{\alpha}(t), t \geq 0$, with one initial progenitor and birth rate $\alpha>0$, with an independent process of the same kind, with 
parameter $\lambda>0$, i.e.

$$
\mathcal{Z}(t):=B_{\alpha}\left(B_{\lambda}(t)\right), \quad t \geq 0
$$

As a consequence of the definition (3.1), the initial number of progenitors of the iterated linear birth process is random, since $\mathcal{Z}(0)=B_{\alpha}(1)$ a.s.

The probability mass function of this process can be written, for any $k \geq 1$, as

$$
\begin{aligned}
q_{k}^{\mathcal{Z}}(t) & :=\operatorname{Pr}\{\mathcal{Z}(t)=k\}=\mathbb{E} \operatorname{Pr}\left\{B_{\alpha}\left(B_{\lambda}(t)\right)=k \mid B_{\lambda}(t)\right\} \\
& =\sum_{j=1}^{\infty} \operatorname{Pr}\left\{B_{\alpha}(j)=k\right\} \operatorname{Pr}\left\{B_{\lambda}(t)=j\right\} \\
& =e^{-\lambda t} \sum_{j=1}^{\infty} e^{-\alpha j}\left(1-e^{-\alpha j}\right)^{k-1}\left(1-e^{-\lambda t}\right)^{j-1} \\
& =e^{-\lambda t-\alpha} \sum_{l=0}^{k-1}\left(\begin{array}{c}
k-1 \\
l
\end{array}\right) \frac{\left(-e^{-\alpha}\right)^{l}}{1-e^{-\alpha(l+1)}\left(1-e^{-\lambda t}\right)}
\end{aligned}
$$

We observe that

$$
\begin{aligned}
& q_{1}^{\mathcal{Z}}(t)=\frac{e^{-\lambda t-\alpha}}{1-e^{-\alpha}\left(1-e^{-\lambda t}\right)}=\mathbb{E} e^{-\alpha B_{\lambda}(t)} \\
& q_{2}^{\mathcal{Z}}(t)=q_{1}^{\mathcal{Z}}(t) \frac{1-e^{-\alpha}}{1-e^{-2 \alpha}\left(1-e^{-\lambda t}\right)} \leq q_{1}^{\mathcal{Z}}(t) .
\end{aligned}
$$

We prove now that the probabilities $q_{k}^{\mathcal{Z}}(t), k \geq 1, t \geq 0$ decrease as $k$ increases, since we have that

$$
\begin{aligned}
q_{k}^{\mathcal{Z}}(t)-q_{k-1}^{\mathcal{Z}}(t) & =e^{-\lambda t-\alpha}\left[\sum_{l=1}^{k-2}\left(\begin{array}{c}
k-2 \\
l-1
\end{array}\right) \frac{\left(-e^{-\alpha}\right)^{l}}{1-e^{-\alpha(l+1)}\left(1-e^{-\lambda t}\right)}+\frac{\left(-e^{-\alpha}\right)^{k-1}}{1-e^{-\alpha k}\left(1-e^{-\lambda t}\right)}\right] \\
& =e^{-\lambda t-\alpha} \sum_{l=1}^{k-1}\left(\begin{array}{c}
k-2 \\
l-1
\end{array}\right) \frac{\left(-e^{-\alpha}\right)^{l}}{1-e^{-\alpha(l+1)}\left(1-e^{-\lambda t}\right)} \\
& =-e^{-\lambda t-2 \alpha} \sum_{m=0}^{\infty}\left(1-e^{-\lambda t}\right)^{m} e^{-2 \alpha m} \sum_{l=0}^{k-2}\left(\begin{array}{c}
k-2 \\
l
\end{array}\right)(-1)^{l} e^{-\alpha l(m+1)} \\
& =-e^{-\lambda t-2 \alpha} \sum_{m=0}^{\infty}\left(1-e^{-\lambda t}\right)^{m} e^{-2 \alpha m}\left(1-e^{-\alpha(m+1)}\right)^{k-2}<0,
\end{aligned}
$$

for any $k \geq 2$.

The sample paths of the iterated birth process display upward jumps of size larger or equal to one. For this reason the analysis of the first-passage time through an arbitrary level $k \geq 2$, i.e.

$$
T_{k}^{\mathcal{Z}}=\inf \{t>0: \mathcal{Z}(t)=k\}
$$

is of a certain importance. Moreover, we shall prove that $\operatorname{Pr}\left\{T_{k}^{\mathcal{Z}}<\infty\right\}$ is strictly less than one, as it happens for the iterated Poisson process (see [12]) and thus any level $k$ can be avoided with positive probability, because of "multiple jumps". We present the explicit distribution of $T_{k}^{\mathcal{Z}}$ in the next theorem.

Theorem 1 The distribution of $T_{k}^{\mathcal{Z}}$ reads

$$
\operatorname{Pr}\left\{T_{k}^{\mathcal{Z}} \in d t\right\} / d t=\lambda e^{-\lambda t-\alpha k} \sum_{j=1}^{\infty} j e^{-\alpha j}\left(1-e^{-\lambda t}\right)^{j-1}\left[\left(e^{\alpha}-e^{-\alpha j}\right)^{k-1}-\left(1-e^{-\alpha j}\right)^{k-1}\right],
$$

for any $k \geq 2,0 \leq t<\infty$. 
Proof. By a conditioning argument we get

$$
\begin{aligned}
\operatorname{Pr}\left\{T_{k}^{\mathcal{Z}} \in d t\right\} & =\mathbb{E} \operatorname{Pr}\left\{T_{k}^{\mathcal{Z}} \in d t \mid B_{\alpha}(1)\right\} \\
& =\sum_{h=1}^{k-1} \operatorname{Pr}\left\{B_{\alpha}\left(B_{\lambda}(t)\right)=k-h, B_{\alpha}\left(B_{\lambda}(t+d t)\right)=k\right\} \\
& =\lambda d t \sum_{j=1}^{\infty} j \sum_{h=1}^{k-1} \operatorname{Pr}\left\{B_{\alpha}\left(B_{\lambda}(t)\right)=k-h, B_{\alpha}\left(B_{\lambda}(t)+1\right)=k \mid B_{\lambda}(t)=j\right\} \operatorname{Pr}\left\{B_{\lambda}(t)=j\right\} \\
& =\lambda d t \sum_{j=1}^{\infty} j \operatorname{Pr}\left\{B_{\lambda}(t)=j\right\} \sum_{h=1}^{k-1} \operatorname{Pr}\left\{B_{\alpha}(j+1)=k \mid B_{\alpha}(j)=k-h\right\} \operatorname{Pr}\left\{B_{\alpha}(j)=k-h\right\} \\
& =\lambda e^{-\lambda t-\alpha k} d t \sum_{j=1}^{\infty} j e^{-\alpha j}\left(1-e^{-\lambda t}\right)^{j-1} \sum_{h=1}^{k-1}\left(\begin{array}{c}
k-1 \\
h
\end{array}\right)\left(e^{\alpha}-1\right)^{h}\left(1-e^{-\alpha j}\right)^{k-h-1},
\end{aligned}
$$

which coincides with 3.2 .

We note that, in the special case $k=2$, we get

$$
\operatorname{Pr}\left\{T_{2}^{\mathcal{Z}} \in d t\right\}=\frac{\lambda e^{-\lambda t-2 \alpha}\left(1-e^{-\alpha}\right)}{\left[1-e^{-\alpha}\left(1-e^{-\lambda t}\right)\right]^{2}} d t=\lambda e^{\lambda t}\left(1-e^{-\alpha}\right)\left[q_{1}^{\mathcal{Z}}(t)\right]^{2} d t
$$

Remark 2 By integrating (3.2), we get that

$$
\operatorname{Pr}\left\{T_{k}^{\mathcal{Z}}<\infty\right\}=e^{-\alpha k} \sum_{j=1}^{\infty} e^{-\alpha j}\left[\left(e^{\alpha}-e^{-\alpha j}\right)^{k-1}-\left(1-e^{-\alpha j}\right)^{k-1}\right],
$$

which can be alternatively written as

$$
\begin{aligned}
\operatorname{Pr}\left\{T_{k}^{\mathcal{Z}}<\infty\right\} & =\sum_{j=2}^{\infty} e^{-\alpha j}\left(1-e^{-\alpha j}\right)^{k-1}-e^{-\alpha k} \sum_{j=1}^{\infty} e^{-\alpha j}\left(1-e^{-\alpha j}\right)^{k-1} \\
& =\left(1-e^{-\alpha k}\right) \sum_{j=2}^{\infty} e^{-\alpha j}\left(1-e^{-\alpha j}\right)^{k-1}-e^{-\alpha k-\alpha}\left(1-e^{-\alpha}\right)^{k-1} \\
& =\left(1-e^{-\alpha k}\right) \sum_{j=2}^{\infty} \operatorname{Pr}\left\{B_{\alpha}(j)=k\right\}-e^{-\alpha k} \operatorname{Pr}\left\{B_{\alpha}(1)=k\right\}
\end{aligned}
$$

In order to analyze some special cases, we supply also the following finite sum form of the hitting probabilities given in (3.3):

$$
\begin{aligned}
\operatorname{Pr}\left\{T_{k}^{\mathcal{Z}}<\infty\right\} & =e^{-\alpha k} \sum_{j=1}^{\infty} e^{-\alpha j} \sum_{r=0}^{k-1}\left(\begin{array}{c}
k-1 \\
r
\end{array}\right)\left[\left(-e^{-\alpha j}\right)^{r} e^{\alpha(k-r-1)}-\left(-e^{-\alpha j}\right)^{r}\right] \\
& =e^{-\alpha k} \sum_{r=0}^{k-2}\left(\begin{array}{c}
k-1 \\
r
\end{array}\right)(-1)^{r} \frac{e^{-\alpha(r+1)}}{1-e^{-\alpha(r+1)}}\left(e^{\alpha(k-r-1)}-1\right) .
\end{aligned}
$$

It is now easy to show that the following relationships hold, for $k=2,3,4$ :

$$
\operatorname{Pr}\left\{T_{4}^{\mathcal{Z}}<\infty\right\}<\operatorname{Pr}\left\{T_{2}^{\mathcal{Z}}<\infty\right\}<\operatorname{Pr}\left\{T_{3}^{\mathcal{Z}}<\infty\right\},
$$


since we have that

$$
\begin{aligned}
& \operatorname{Pr}\left\{T_{2}^{\mathcal{Z}}<\infty\right\}=e^{-2 \alpha} \\
& \operatorname{Pr}\left\{T_{3}^{\mathcal{Z}}<\infty\right\}=e^{-2 \alpha}\left[1+e^{-\alpha} \frac{1-e^{-\alpha}}{1+e^{-\alpha}}\right] \\
& \operatorname{Pr}\left\{T_{4}^{\mathcal{Z}}<\infty\right\}=e^{-2 \alpha}\left[1-e^{-\alpha} \frac{\left(1-e^{-\alpha}\right)\left(1+e^{-3 \alpha}\right)}{1+e^{-\alpha}+e^{-2 \alpha}}\right] .
\end{aligned}
$$

The following figures (produced by the software $R$ ) describe the behavior of the probabilities (3.5), for different values of $\alpha$. We remark that the scales are not the same in different figures.

Figure 1: Hitting times $\operatorname{Pr}\left\{T_{k}^{\mathcal{Z}}<\infty\right\}$

$\alpha=1 / 4$

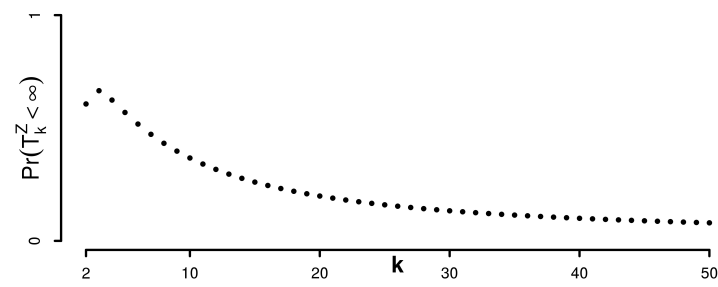

$\alpha=2$

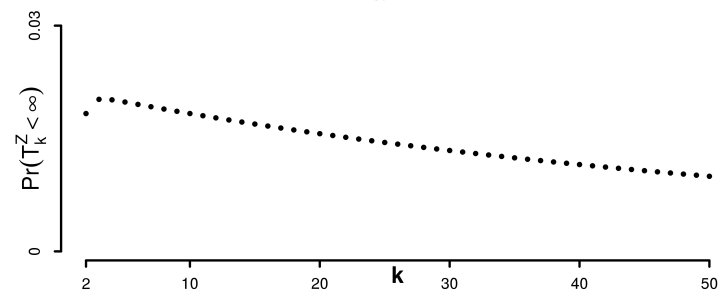

$\alpha=1 / 2$

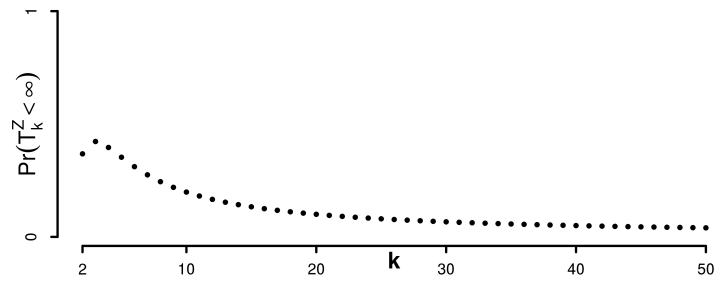

$\alpha=3$

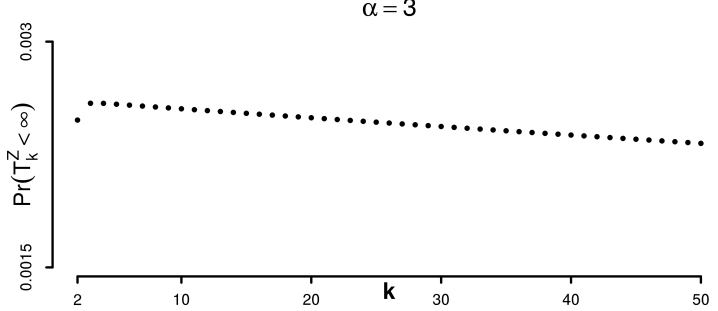

The figures above show that the probabilities $\operatorname{Pr}\left\{T_{k}^{\mathcal{Z}}<\infty\right\}$, for $k>3$, are decreasing functions which, for $0<\alpha<1$, vanish more rapidly than for $\alpha>1$.

The rate of decreasing is slow, for all $\alpha$, since, from 3.4 , we get that

$$
\begin{aligned}
\sum_{k=2}^{\infty} \operatorname{Pr}\left\{T_{k}^{\mathcal{Z}}<\infty\right\} & =\sum_{j=2}^{\infty} e^{-\alpha j} \sum_{k=2}^{\infty}\left(1-e^{-\alpha j}\right)^{k-1}-\sum_{j=1}^{\infty} e^{-\alpha(j+1)} \sum_{k=2}^{\infty}\left(e^{-\alpha}-e^{-\alpha(j+1)}\right)^{k-1} \\
& =\sum_{j=2}^{\infty}\left(1-e^{-\alpha j}\right)-\sum_{j=1}^{\infty} e^{-\alpha(j+1)-\alpha} \frac{1-e^{-\alpha j}}{1-e^{-\alpha}+e^{-\alpha(j+1)}} \\
& =\sum_{j=2}^{\infty} \frac{\left(1-e^{-\alpha j}\right)\left[1-e^{-\alpha}-e^{-\alpha(j+1)}-e^{-\alpha(j+1)-\alpha}\right]}{1-e^{-\alpha}+e^{-\alpha(j+1)}-\frac{e^{-3 \alpha}\left(1-e^{-\alpha}\right)}{1-e^{-\alpha}+e^{-2 \alpha}}} \\
& =\left(1-e^{-\alpha}\right)\left\{\sum_{j=2}^{\infty} \frac{\left(1-e^{-\alpha j}\right)\left[1+e^{-\alpha(j+1)}\right]}{1-e^{-\alpha}+e^{-\alpha(j+1)}}-\frac{e^{-3 \alpha}}{1-e^{-\alpha}+e^{-2 \alpha}}\right\}=\infty
\end{aligned}
$$

since

$$
\lim _{j \rightarrow \infty} \frac{\left(1-e^{-\alpha j}\right)\left[1+e^{-\alpha(j+1)}\right]}{1-e^{-\alpha}+e^{-\alpha(j+1)}}=\frac{1}{1-e^{-\alpha}}
$$




\section{Linear birth process at Poisson times}

We now pass to the analysis of the process defined as

$$
\mathcal{X}(t):=B_{\alpha}\left(N_{\lambda}(t)\right), \quad t \geq 0
$$

obtained as a composition of a linear birth process $B_{\alpha}$ with an independent homogeneous Poisson process $N_{\lambda}(t), t \geq 0$, with rate $\lambda>0$. The process $\mathcal{X}$ can be regarded as a linear birth process randomly sampled at Poisson times. We first note that, for $k \geq n_{0}$,

$$
\begin{aligned}
q_{k, n_{0}}^{\mathcal{X}}(t) & :=\operatorname{Pr}\left\{\mathcal{X}(t)=k \mid \mathcal{X}(0)=n_{0}\right\} \\
& =e^{-\lambda t} \sum_{j=0}^{\infty}\left(\begin{array}{c}
k-1 \\
k-n_{0}
\end{array}\right) e^{-\alpha j n_{0}}\left(1-e^{-\alpha j}\right)^{k-n_{0}} \frac{(\lambda t)^{j}}{j !} \\
& =\sum_{l=0}^{k-n_{0}}\left(\begin{array}{c}
k-n_{0} \\
l
\end{array}\right)(-1)^{l} \exp \left\{\lambda t\left[1-e^{-\alpha\left(n_{0}+l\right)}\right]\right\} .
\end{aligned}
$$

In particular, for $k=n_{0}$, we get

$$
q_{n_{0}, n_{0}}^{\mathcal{X}}(t)=e^{-\lambda t\left(1-e^{-\alpha n_{0}}\right)}
$$

and thus the waiting time for the appearance of the first offspring is exponentially distributed with parameter $\lambda\left(1-e^{-\alpha n_{0}}\right)$. It is easy to show that

$$
\mathbb{E} \mathcal{X}(t)=n_{0} e^{\lambda t\left(e^{\alpha}-1\right)} .
$$

The variance of $\mathcal{X}$ can be obtained as follows

$$
\begin{aligned}
\operatorname{Var} \mathcal{X}(t) & =\operatorname{Var} \mathbb{E}\left[B_{\alpha}\left(N_{\lambda}(t)\right) \mid N_{\lambda}(t)\right]+\mathbb{E} \operatorname{Var}\left[B_{\alpha}\left(N_{\lambda}(t)\right) \mid N_{\lambda}(t)\right] \\
& =\operatorname{Var}\left[n_{0} e^{\alpha N_{\lambda}(t)}\right]+\mathbb{E}\left[n_{0} e^{2 \alpha N_{\lambda}(t)}-n_{0} e^{\alpha N_{\lambda}(t)}\right] \\
& =n_{0}\left(n_{0}+1\right) e^{\lambda t\left(e^{2 \alpha}-1\right)}-n_{0}^{2} e^{\lambda t\left(e^{\alpha}-1\right)}-n_{0} e^{2 \lambda t\left(e^{\alpha}-1\right)} .
\end{aligned}
$$

For the sake of simplicity, from now on, we assume $n_{0}=1$ and denote for brevity $q_{k, 1}^{\mathcal{X}}$ as $q_{k}^{\mathcal{X}}$. The factorial moments of $\mathcal{X}$ can be evaluated as follows:

$$
\begin{aligned}
& \mathbb{E}\{\mathcal{X}(t)[\mathcal{X}(t)-1] \ldots[\mathcal{X}(t)-r+1] \mid \mathcal{X}(t)=1\} \\
= & \sum_{k=r}^{\infty} k(k-1) \ldots(k-r+1) q_{k}^{\mathcal{X}}(t) \\
= & {[\text { by } 4.1)] } \\
= & r ! e^{-\lambda t} \sum_{k=r}^{\infty}\left(\begin{array}{c}
k \\
r
\end{array}\right) \sum_{j=0}^{\infty} e^{-\alpha j}\left(1-e^{-\alpha j}\right)^{k-1} \frac{(\lambda t)^{j}}{j !} \\
= & r ! e^{-\lambda t} \sum_{j=0}^{\infty} e^{-\alpha j} \frac{(\lambda t)^{j}}{j !} \sum_{l=0}^{\infty}\left(\begin{array}{c}
r+l \\
l
\end{array}\right)\left(1-e^{-\alpha j}\right)^{r+l-1} \\
= & r ! e^{-\lambda t} \sum_{j=0}^{\infty} e^{-\alpha j} \frac{(\lambda t)^{j}}{j !} \frac{\left(1-e^{-\alpha j}\right)^{r-1}}{\left[1-\left(1-e^{-\alpha j}\right)\right]^{r+1}}
\end{aligned}
$$




$$
\begin{aligned}
& =r ! e^{-\lambda t} \sum_{j=0}^{\infty} \frac{\left(\lambda t e^{\alpha r}\right)^{j}}{j !}\left(1-e^{-\alpha j}\right)^{r-1} \\
& =r ! e^{-\lambda t} \sum_{j=0}^{\infty} \frac{\left(\lambda t e^{\alpha r}\right)^{j}}{j !} \sum_{m=0}^{r-1}\left(\begin{array}{c}
r-1 \\
m
\end{array}\right)(-1)^{m} e^{-\alpha j m} \\
& =r ! \sum_{m=0}^{r-1}\left(\begin{array}{c}
r-1 \\
m
\end{array}\right)(-1)^{m} \exp \left\{-\lambda t\left(1-e^{\alpha(r-m)}\right)\right\} .
\end{aligned}
$$

By using result (4.5) we can check formulas (4.3) and (4.4), by means of independent calculations.

Our main interest here is to study the distribution of the first-passage time for an arbitrary level $k$, i.e.

$$
T_{k}^{\mathcal{X}}=\inf \{t>0: \mathcal{X}(t)=k\}
$$

for $k \geq 2$. In the next theorem we derive the following explicit distribution of $T_{k}^{\mathcal{X}}$.

Theorem 3 For $t \geq 0$ and $k \geq 2$ we have that

$$
\operatorname{Pr}\left\{T_{k}^{\mathcal{X}} \in d t \mid \mathcal{X}(0)=1\right\} / d t=\lambda e^{-\alpha k} \sum_{l=0}^{k-2}(-1)^{l}\left(\begin{array}{c}
k-1 \\
l
\end{array}\right)\left(e^{\alpha(k-1-l)}-1\right) \exp \left\{-\lambda t\left[1-e^{-\alpha(l+1)}\right]\right\}
$$

Proof. We start by considering that

$$
\begin{aligned}
& \operatorname{Pr}\left\{T_{k}^{\mathcal{X}} \in d t \mid \mathcal{X}(0)=1\right\} \\
& =\sum_{h=1}^{k-1} \operatorname{Pr}\left\{B_{\alpha}\left(N_{\lambda}(t)\right)=k-h, B_{\alpha}\left(N_{\lambda}(t+d t)\right)=k\right\} \\
& =\sum_{h=1}^{k-1} \operatorname{Pr}\left\{B_{\alpha}\left(N_{\lambda}(t)\right)=k-h, B_{\alpha}\left(N_{\lambda}(t)+d N_{\lambda}(t)\right)=k\right\} \\
& =\lambda d t \sum_{j=0}^{\infty} \sum_{h=1}^{k-1} \operatorname{Pr}\left\{B_{\alpha}\left(N_{\lambda}(t)\right)=k-h, B_{\alpha}\left(N_{\lambda}(t)+1\right)=k \mid N_{\lambda}(t)=j\right\} \operatorname{Pr}\left\{N_{\lambda}(t)=j\right\} \\
& =\lambda e^{-\lambda t} d t \sum_{j=0}^{\infty} \frac{(\lambda t)^{j}}{j !} \sum_{h=1}^{k-1} \operatorname{Pr}\left\{B_{\alpha}(j)=k-h, B_{\alpha}(j+1)=k\right\} \\
& =\lambda e^{-\lambda t} d t \sum_{j=0}^{\infty} \frac{(\lambda t)^{j}}{j !} \sum_{h=1}^{k-1} e^{-\alpha j}\left(1-e^{-\alpha j}\right)^{k-h-1}\left(\begin{array}{c}
k-1 \\
h
\end{array}\right) e^{-\alpha(k-h)}\left(1-e^{-\alpha}\right)^{h} \\
& =\lambda e^{-\lambda t-\alpha k} d t \sum_{h=1}^{k-1}\left(\begin{array}{c}
k-1 \\
h
\end{array}\right)\left(e^{\alpha}-1\right)^{h} \sum_{l=0}^{k-h-1}\left(\begin{array}{c}
k-h-1 \\
l
\end{array}\right)(-1)^{l} \sum_{j=0}^{\infty} \frac{\left(\lambda t e^{-\alpha(l+1)}\right)^{j}}{j !} \\
& =\lambda e^{-\lambda t-\alpha k} d t \sum_{h=1}^{k-1}\left(\begin{array}{c}
k-1 \\
h
\end{array}\right)\left(e^{\alpha}-1\right)^{h} \sum_{l=0}^{k-h-1}\left(\begin{array}{c}
k-h-1 \\
l
\end{array}\right)(-1)^{l} \exp \left\{\lambda t e^{-\alpha(l+1)}\right\} \\
& =\lambda e^{-\lambda t-\alpha k} d t \sum_{l=0}^{k-2}(-1)^{l} \exp \left\{\lambda t e^{-\alpha(l+1)}\right\} \sum_{h=1}^{k-l-1}\left(\begin{array}{c}
k-1 \\
h
\end{array}\right)\left(\begin{array}{c}
k-h-1 \\
l
\end{array}\right)\left(e^{\alpha}-1\right)^{h} \\
& =\lambda e^{-\alpha k} d t \sum_{l=0}^{k-2}\left(\begin{array}{c}
k-1 \\
l
\end{array}\right)(-1)^{l} \exp \left\{-\lambda t\left(1-e^{-\alpha(l+1)}\right)\right\} \sum_{h=1}^{k-l-1}\left(\begin{array}{c}
k-l-1 \\
h
\end{array}\right)\left(e^{\alpha}-1\right)^{h},
\end{aligned}
$$

which coincides with 4.6 . 
Remark 4 In the particular case $k=2$, formula (4.6) reduces to

$$
\begin{aligned}
\operatorname{Pr}\left\{T_{2}^{\mathcal{X}} \in d t \mid \mathcal{X}(0)=1\right\} / d t & =\lambda e^{-\alpha}\left(1-e^{-\alpha}\right) e^{-\lambda t\left(1-e^{-\alpha}\right)} \\
& =e^{-\alpha} \operatorname{Pr}\{Z \in d t\}
\end{aligned}
$$

where $Z$ is an exponential r.v. with parameter $\lambda\left(1-e^{-\alpha}\right)$ and this clearly shows that for the firstpassage time through the level $k=2$, the following result holds

$$
\operatorname{Pr}\left\{T_{2}^{\mathcal{X}}<\infty \mid \mathcal{X}(0)=1\right\}=e^{-\alpha}<1
$$

Remark 5 For any $k \geq 2$, by integrating formula (4.6), we get

$$
\begin{aligned}
& \operatorname{Pr}\left\{T_{k}^{\mathcal{X}}<\infty \mid \mathcal{X}(0)=1\right\} \\
& =e^{-\alpha k} \sum_{l=0}^{k-2}(-1)^{l}\left(\begin{array}{c}
k-1 \\
l
\end{array}\right) \frac{e^{\alpha(k-1-l)}-1}{1-e^{-\alpha(l+1)}} \\
& =e^{-\alpha k} \sum_{l=0}^{k-2}(-1)^{l}\left(\begin{array}{c}
k-1 \\
l
\end{array}\right)\left[e^{\alpha(k-1-l)}-1\right] \sum_{m=0}^{\infty} e^{-\alpha(l+1) m} \\
& =e^{-\alpha k} \sum_{m=0}^{\infty} e^{-\alpha m} \sum_{l=0}^{k-2}(-1)^{l}\left(\begin{array}{c}
k-1 \\
l
\end{array}\right)\left[e^{\alpha(k-1)-\alpha l(m+1)}-e^{-\alpha l m}\right] \\
& =\sum_{m=0}^{\infty} e^{-\alpha(m+1)}\left[\left(1-e^{-\alpha(m+1)}\right)^{k-1}-(-1)^{k-1} e^{-\alpha(m+1)(k-1)}\right]+ \\
& -e^{-\alpha k} \sum_{m=0}^{\infty} e^{-\alpha m}\left[\left(1-e^{-\alpha m}\right)^{k-1}-(-1)^{k-1} e^{-\alpha m(k-1)}\right] \\
& =\sum_{m=0}^{\infty}\left[e^{-\alpha(m+1)}\left(1-e^{-\alpha(m+1)}\right)^{k-1}-e^{-\alpha k} e^{-\alpha m}\left(1-e^{-\alpha m}\right)^{k-1}\right] \\
& =\left(1-e^{-\alpha k}\right) \sum_{m=1}^{\infty} e^{-\alpha m}\left(1-e^{-\alpha m}\right)^{k-1} \\
& =\left(1-e^{-\alpha k}\right) \sum_{m=1}^{\infty} \operatorname{Pr}\left\{B_{\alpha}(m)=k\right\} .
\end{aligned}
$$

As in the case of the iterated birth process, the hitting probabilities (4.8) are not affected by the parameter $\lambda$ of the inner process $\left(N_{\lambda}\right.$, in this case). Moreover, we remark that the relationship given in the last line is equal to the analogous one presented in (3.4) (except for the additional term in (3.4), which is due to the different starting point of the birth process with respect to the Poisson one):

$$
\operatorname{Pr}\left\{T_{k}^{\mathcal{X}}<\infty \mid \mathcal{X}(0)=1\right\}-\operatorname{Pr}\left\{T_{k}^{\mathcal{Z}}<\infty\right\}=\operatorname{Pr}\left\{B_{\alpha}(1)=k\right\}
$$

for any $k \geq 2$. Thus, for the iterated linear birth process, the probability of reaching any level $k$ in a finite time is strictly smaller than the corresponding probability for $B_{\alpha}\left(N_{\lambda}(t)\right), t>0$. This difference decreases monotonically with $k$.

Remark 6 For $k=2$ the first line of formula (4.8) reduces to (4.7). In the special case $k=3$ we 
have that

$$
\begin{aligned}
& \operatorname{Pr}\left\{T_{3}^{\mathcal{X}}<\infty \mid \mathcal{X}(0)=1\right\} \\
= & e^{-3 \alpha} \frac{e^{\alpha}-1}{1-e^{-\alpha}}\left[e^{\alpha}+1-\frac{2}{1+e^{-\alpha}}\right] \\
= & e^{-\alpha} \frac{1+e^{-2 \alpha}}{1+e^{-\alpha}} \\
= & \operatorname{Pr}\left\{T_{2}^{\mathcal{X}}<\infty \mid \mathcal{X}(0)=1\right\} \frac{1+e^{-2 \alpha}}{1+e^{-\alpha}}<\operatorname{Pr}\left\{T_{2}^{\mathcal{X}}<\infty \mid \mathcal{X}(0)=1\right\}<1 .
\end{aligned}
$$

We show now that, for any $k \geq 2$ the distribution of the first-passage time for the level $k$ is monotonically decreasing: we present the following heuristic argument

$$
\begin{aligned}
& \operatorname{Pr}\left\{T_{k}^{\mathcal{X}}<\infty \mid \mathcal{X}(0)=1\right\}-\operatorname{Pr}\left\{T_{k-1}^{\mathcal{X}}<\infty \mid \mathcal{X}(0)=1\right\} \\
= & \left(1-e^{-\alpha k}\right) \sum_{m=0}^{\infty} e^{-\alpha m}\left(1-e^{-\alpha m}\right)^{k-1}-\left(1-e^{-\alpha(k-1)}\right) \sum_{m=0}^{\infty} e^{-\alpha m}\left(1-e^{-\alpha m}\right)^{k-2} \\
\simeq & \left(1-e^{-\alpha k}\right) \int_{0}^{+\infty} e^{-\alpha x}\left(1-e^{-\alpha x}\right)^{k-1} d x-\left(1-e^{-\alpha(k-1)}\right) \int_{0}^{+\infty} e^{-\alpha x}\left(1-e^{-\alpha x}\right)^{k-2} d x \\
= & \frac{1-e^{-\alpha k}}{\alpha k}-\frac{1-e^{-\alpha(k-1)}}{\alpha(k-1)} \\
= & \frac{1-e^{-\alpha}}{\alpha}\left[\frac{1+\ldots+e^{-\alpha(k-1)}}{k}-\frac{1+\ldots+e^{-\alpha(k-2)}}{k-1}\right] \\
= & \frac{\left(1-e^{-\alpha}\right)}{\alpha}\left\{\left(1+\ldots+e^{-\alpha(k-2)}\right)\left[\frac{1}{k}-\frac{1}{k-1}\right]+\frac{e^{-\alpha(k-1)}}{k}\right\} \\
\leq & \frac{\left(1-e^{-\alpha}\right)}{\alpha}\left[-\frac{e^{-\alpha(k-2)}}{k(k-1)}(k-1)+\frac{e^{-\alpha(k-1)}}{k}\right]<0,
\end{aligned}
$$

for $k>2$.

The plots of Fig.2 confirm the decreasing structure of the probabilities $\operatorname{Pr}\left\{T_{k}^{\mathcal{X}}<\infty \mid \mathcal{X}(0)=1\right\}$.

Remark 7 From formula (4.8) we derive the following equality

$$
\begin{aligned}
g_{k}^{\mathcal{X}}-g_{k-1}^{\mathcal{X}} & :=\frac{\operatorname{Pr}\left\{T_{k}^{\mathcal{X}}<\infty \mid \mathcal{X}(0)=1\right\}}{1-e^{-\alpha k}}-\frac{\operatorname{Pr}\left\{T_{k-1}^{\mathcal{X}}<\infty \mid \mathcal{X}(0)=1\right\}}{1-e^{-\alpha(k-1)}} \\
& =-\sum_{m=1}^{\infty} e^{-2 \alpha m}\left(1-e^{-\alpha m}\right)^{k-2}<0 .
\end{aligned}
$$

It is clear from (4.9) that, for large values of $k$, we get

$$
\operatorname{Pr}\left\{T_{k}^{\mathcal{X}}<\infty \mid \mathcal{X}(0)=1\right\} \approx \operatorname{Pr}\left\{T_{k-1}^{\mathcal{X}}<\infty \mid \mathcal{X}(0)=1\right\}
$$

and thus the hitting probabilities slowly change with $k$ as Fig. 2 confirms.

By applying formula (4.9) we can prove also the following relationship

$$
\begin{aligned}
& \frac{\operatorname{Pr}\left\{T_{k}^{\mathcal{X}}<\infty \mid \mathcal{X}(0)=1\right\}}{1-e^{-\alpha k}}-\frac{\operatorname{Pr}\left\{T_{k-2}^{\mathcal{X}}<\infty \mid \mathcal{X}(0)=1\right\}}{1-e^{-\alpha(k-2)}} \\
= & -\sum_{m=1}^{\infty} e^{-2 \alpha m}\left(1-e^{-\alpha m}\right)^{k-3}\left(2-e^{-\alpha m}\right) \\
= & 2\left[\frac{\operatorname{Pr}\left\{T_{k-1}^{\mathcal{X}}<\infty \mid \mathcal{X}(0)=1\right\}}{1-e^{-\alpha(k-1)}}-\frac{\operatorname{Pr}\left\{T_{k-2}^{\mathcal{X}}<\infty \mid \mathcal{X}(0)=1\right\}}{1-e^{-\alpha(k-2)}}\right]+\sum_{m=1}^{\infty} e^{-3 \alpha m}\left(1-e^{-\alpha m}\right)^{k-3} .
\end{aligned}
$$


Figure 2: Hitting times $\operatorname{Pr}\left\{T_{k}^{\mathcal{X}}<\infty\right\}$
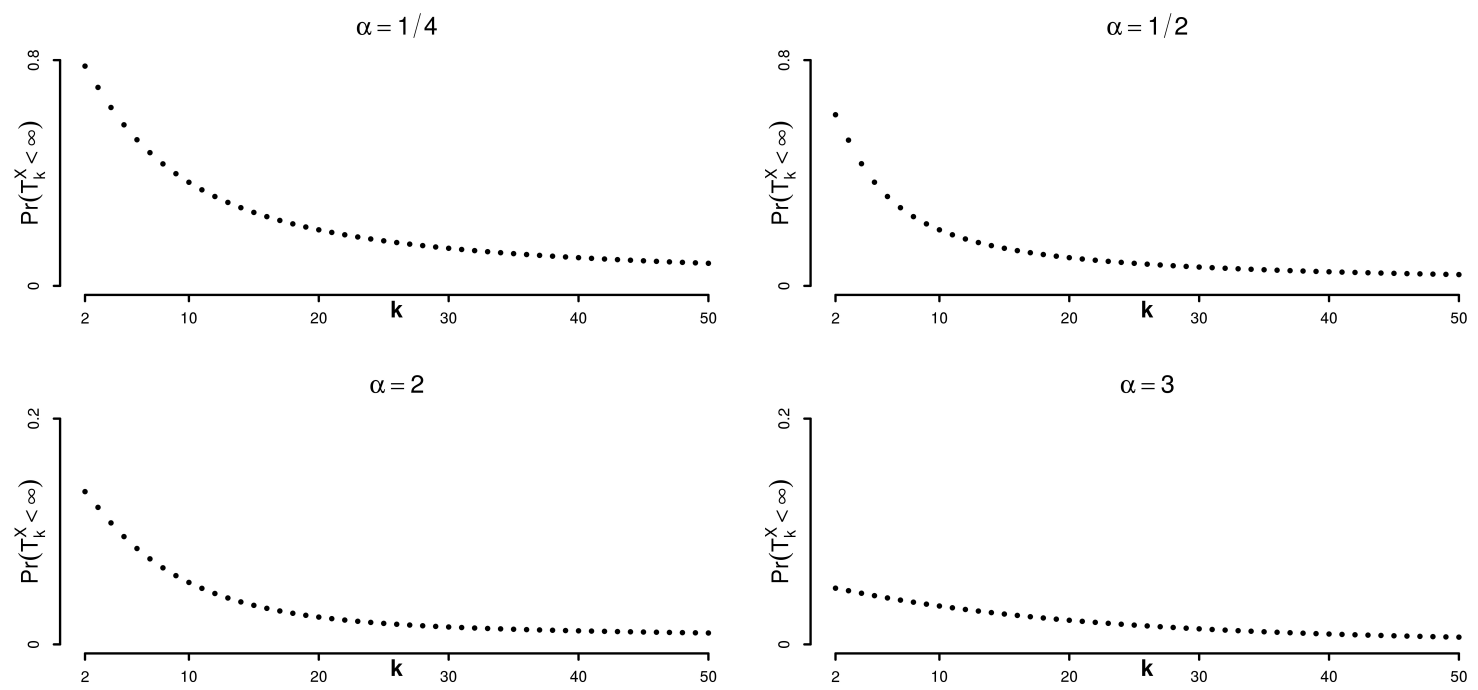

Formula (4.10) shows that, for large values of $k$,

$$
g_{k}^{\mathcal{X}}-g_{k-2}^{\mathcal{X}} \simeq 2\left(g_{k-1}^{\mathcal{X}}-g_{k-2}^{\mathcal{X}}\right) .
$$

\section{Death processes at Poisson times}

We here consider linear and sublinear death processes at Poisson times. The linear death process, with initial population of $n_{0}$ individuals and death rate $\mu>0$, has a binomial distribution, i.e.

$$
p_{k}^{D}:=\operatorname{Pr}\left\{D_{\mu}(t)=k \mid D_{\mu}(0)=n_{0}\right\}=\left(\begin{array}{c}
n_{0} \\
k
\end{array}\right) e^{-\mu t k}\left(1-e^{-\mu t}\right)^{n_{0}-k}, \quad 0 \leq k \leq n_{0} .
$$

The probabilities (5.1) satisfy the difference-differential equations

$$
\left\{\begin{array}{l}
\frac{d}{d t} p_{k}^{D}(t)=-\mu k p_{k}^{D}(t)+\mu(k+1) p_{k+1}^{D}(t) \quad \text { for } 0 \leq k \leq n_{0}-1 \\
\frac{d}{d t} p_{n_{0}}^{D}(t)=-\mu n_{0} p_{n_{0}}^{D}(t) \quad \text { for } k=n_{0}
\end{array}\right.
$$

with $p_{k}^{D}(0)=1_{\left\{k=n_{0}\right\}}$.

Moreover, we denote the sublinear death process, with death rate $\mu>0$, as $\widetilde{D}(t), t \geq 0$ and its distribution is given by

$$
p_{k}^{\widetilde{D}}(t):=\operatorname{Pr}\left\{\widetilde{D}_{\mu}(t)=k \mid \widetilde{D}_{\mu}(0)=n_{0}\right\}=\left\{\begin{array}{l}
e^{-\mu t}\left(1-e^{-\mu t}\right)^{n_{0}-k}, \quad 1 \leq k \leq n_{0} \\
\left(1-e^{-\mu t}\right)^{n_{0}}, \quad k=0 .
\end{array}\right.
$$

The probabilities $p_{k}^{\widetilde{D}}$ satisfy the following difference-differential equations

$$
\left\{\begin{array}{l}
\frac{d}{d t} p_{k}^{\widetilde{D}}(t)=-\mu\left(n_{0}-k+1\right) p_{k}^{\widetilde{D}}(t)+\mu\left(n_{0}-k\right) p_{k+1}^{\widetilde{D}}(t) \quad 1 \leq k \leq n_{0} \\
\frac{d}{d t} p_{0}^{\widetilde{D}}(t)=\mu n_{0} p_{1}^{\widetilde{D}}(t) \quad k=0
\end{array},\right.
$$

with $p_{k}^{\widetilde{D}}(0)=1_{\left\{k=n_{0}\right\}}$. In order to catch the probabilistic mechanism underlying 5.3 we should consider that

$$
\operatorname{Pr}\left\{\widetilde{D}_{\mu}(t, t+d t)-\widetilde{D}_{\mu}(t)=-1 \mid \widetilde{D}_{\mu}(t)=k\right\}=\mu\left(n_{0}-k+1\right) d t+o(d t), \quad 1 \leq k \leq n_{0} .
$$


We note that in the linear death process the probability of cancellation of an individual in $[t, t+d t)$ is proportional to the number of existing components at time $t$. On the other hand, in the sublinear case, this probability is proportional to the number of deaths recorded up to time $t$. This property makes the sublinear process more suitable for describing epidemics and the diffusion of rumors.

The expected value of $\widetilde{D}(t), t \geq 0$ can be evaluated as follows

$$
\begin{aligned}
\mathbb{E} \widetilde{D}(t) & =e^{-\mu t} \sum_{k=0}^{n_{0}}\left(n_{0}-k\right)\left(1-e^{-\mu t}\right)^{k} \\
& =n_{0}\left[1-\left(1-e^{-\mu t}\right)^{n_{0}+1}\right]-\frac{1}{\mu}\left(1-e^{-\mu t}\right) \frac{d}{d t}\left[\sum_{k=0}^{n_{0}}\left(1-e^{-\mu t}\right)^{k}\right] \\
& =n_{0}\left[1-\left(1-e^{-\mu t}\right)^{n_{0}+1}\right]-\left(1-e^{-\mu t}\right)\left[e^{\mu t}-e^{\mu t}\left(1-e^{-\mu t}\right)^{n_{0}+1}-\left(n_{0}+1\right)\left(1-e^{-\mu t}\right)^{n_{0}}\right] \\
& =n_{0}+\left(1-e^{-\mu t}\right)^{n_{0}+1}+e^{\mu t}\left(1-e^{-\mu t}\right)^{n_{0}+2}+1-e^{\mu t} \\
& =n_{0}+1-e^{\mu t}\left[1-\left(1-e^{-\mu t}\right)^{n_{0}+1}\right], \quad n_{0} \geq 1 .
\end{aligned}
$$

It is easy to check that $\lim _{t \rightarrow+\infty} \mathbb{E} \widetilde{D}(t)=0$, as expected. For the linear death process, the hitting time of the $k$-th event, i.e.

$$
T_{k}^{D}=\inf \left\{s>0: D_{\mu}(s)=k\right\}
$$

has the following distribution

$$
\operatorname{Pr}\left\{T_{k}^{D} \in d t\right\}=\left(\begin{array}{c}
n_{0} \\
k+1
\end{array}\right) e^{-\mu t(k+1)}\left(1-e^{-\mu t}\right)^{n_{0}-(k+1)} \mu(k+1) d t, \quad t \geq 0,0 \leq k \leq n_{0} .
$$

Thus we get

$$
\operatorname{Pr}\left\{T_{k}^{D}<\infty\right\}=1,
$$

for any $k$. On the other hand, in the sublinear case, the distribution of

$$
T_{k}^{\widetilde{D}}=\inf \left\{s>0: \widetilde{D}_{\mu}(s)=k\right\}
$$

reads

$$
\operatorname{Pr}\left\{T_{k}^{\widetilde{D}} \in d t\right\}=e^{-\mu t}\left(1-e^{-\mu t}\right)^{n_{0}-(k+1)} \mu\left(n_{0}-k\right) d t, \quad t \geq 0,0 \leq k \leq n_{0} .
$$

Moreover we get

$$
\begin{aligned}
& \operatorname{Pr}\left\{T_{k}^{\widetilde{D}}<\infty\right\}=\int_{0}^{+\infty} \operatorname{Pr}\left\{T_{k}^{\widetilde{D}} \in d t\right\} d t \\
= & \left(n_{0}-k\right) \sum_{r=0}^{n_{0}-k-1}\left(\begin{array}{c}
n_{0}-k-1 \\
r
\end{array}\right)(-1)^{r} \int_{0}^{+\infty} e^{-\mu t(r+1)} d t \\
= & \sum_{r=0}^{n_{0}-k-1}\left(\begin{array}{c}
n_{0}-k \\
r+1
\end{array}\right)(-1)^{r}=1,
\end{aligned}
$$

for any $k$.

\subsection{Linear subordinated death process}

Let

$$
\mathcal{Y}(t):=D_{\mu}\left(N_{\lambda}(t)\right), \quad t \geq 0,
$$


where $D_{\mu}$ is a linear death process with parameter $\mu$ and $N_{\lambda}$ is an independent homogeneous Poisson process with parameter $\lambda$. Then, for $0 \leq k \leq n_{0}$, the probability mass function reads

$$
\begin{aligned}
q_{k}^{\mathcal{Y}}(t) & :=\operatorname{Pr}\left\{\mathcal{Y}(t)=k \mid \mathcal{Y}(0)=n_{0}\right\} \\
& =\sum_{l=0}^{\infty} \operatorname{Pr}\left\{D_{\mu}(l)=k \mid D_{\mu}(0)=n_{0}\right\} \operatorname{Pr}\left\{N_{\lambda}(t)=l\right\} \\
& =\left(\begin{array}{c}
n_{0} \\
k
\end{array}\right) \sum_{j=0}^{n_{0}-k}\left(\begin{array}{c}
n_{0}-k \\
j
\end{array}\right)(-1)^{j} \exp \left\{-\lambda t\left(1-e^{-\mu(k+j)}\right)\right\} .
\end{aligned}
$$

Since

$$
q_{n_{0}}^{\mathcal{Y}}(t)=e^{-\lambda t\left(1-e^{-\mu n_{0}}\right)},
$$

the waiting time of the first death of $\mathcal{Y}(t)$ is exponentially distributed with parameter $\lambda\left(1-e^{-\mu n_{0}}\right)$ and has the same form of the corresponding probability of the subordinated linear birth process, which was given in formula (4.2). The probability generating function of $\mathcal{Y}(t)$ can be written as follows:

$$
\begin{aligned}
G^{\mathcal{Y}}(u, t) & =e^{-\lambda t} \sum_{l=0}^{\infty} \frac{(\lambda t)^{l}}{l !}\left[1-e^{-\mu l}(1-u)\right]^{n_{0}} \\
& =\sum_{m=0}^{n_{0}}\left(\begin{array}{c}
n_{0} \\
m
\end{array}\right)(-1)^{m}(1-u)^{m} \exp \left\{-\lambda t\left(1-e^{-\mu m}\right\} .\right.
\end{aligned}
$$

The mean value and variance are respectively given by

$$
\mathbb{E} \mathcal{Y}(t)=n_{0} e^{\lambda t\left(e^{-\mu}-1\right)}
$$

and

$$
\begin{aligned}
\operatorname{Var} \mathcal{Y}(t) & =\operatorname{Var} \mathbb{E}\left[D_{\mu}\left(N_{\lambda}(t)\right) \mid N_{\lambda}(t)\right]+\mathbb{E} \operatorname{Var}\left[D_{\mu}\left(N_{\lambda}(t)\right) \mid N_{\lambda}(t)\right] \\
& =\operatorname{Var}\left[n_{0} e^{-\mu N_{\lambda}(t)}\right]+\mathbb{E}\left[n_{0} e^{-\mu N_{\lambda}(t)}\left(1-e^{-\mu N_{\lambda}(t)}\right)\right] \\
& =n_{0}\left(n_{0}-1\right) e^{\lambda t\left(e^{-2 \mu}-1\right)}-n_{0}^{2} e^{2 \lambda t\left(e^{-\mu}-1\right)}+n_{0} e^{\lambda t\left(e^{-\mu}-1\right)}
\end{aligned}
$$

We are interested now in the differential equation satisfied by the distribution of the process $\mathcal{Y}(t), t>0$. As a preliminary result we prove the following lemma.

Lemma 8 The probability generating function given in (5.8) satisfies the following initial-value problem:

$$
\left\{\begin{array}{l}
\frac{\partial}{\partial t} G^{\mathcal{Y}}(u, t)=-\lambda\left[1-e^{(1-u)\left(1-e^{-\mu}\right) \partial_{u}}\right] G^{\mathcal{Y}}(u, t), \quad t, u \geq 0, \\
G^{\mathcal{Y}}(u, 0)=u^{n_{0}}
\end{array}\right.
$$

where $e^{a \partial_{u}}:=\sum_{k=0}^{\infty} \frac{a^{k} \partial_{u}^{k}}{k !}$ is the shift operator.

Proof. By considering the equation governing the state probabilities of the Poisson process, we can 
write

$$
\begin{aligned}
\frac{\partial}{\partial t} G^{\mathcal{Y}}(u, t) & =\sum_{l=0}^{\infty} \frac{\partial}{\partial t} \operatorname{Pr}\left\{N_{\lambda}(t)=l\right\} G^{D}(u, l) \\
& =-\lambda \sum_{l=0}^{\infty} \operatorname{Pr}\left\{N_{\lambda}(t)=l\right\} G^{D}(u, l)+\lambda \sum_{l=1}^{\infty} \operatorname{Pr}\left\{N_{\lambda}(t)=l-1\right\} G^{D}(u, l) \\
& =-\lambda G^{\mathcal{Y}}(u, t)+\lambda \sum_{r=0}^{\infty} \operatorname{Pr}\left\{N_{\lambda}(t)=r\right\} G^{D}(u, r+1) \\
& =-\lambda G^{\mathcal{Y}}(u, t)+\lambda \sum_{r=0}^{\infty} \operatorname{Pr}\left\{N_{\lambda}(t)=r\right\} e^{\partial_{r}} G^{D}(u, r)
\end{aligned}
$$

where $G^{D}$ is the probability generating function of $D$. Since, for any $t \geq 0$,

$$
\begin{aligned}
\frac{\partial}{\partial t} G^{D}(u, t)= & \mu(1-u) \frac{\partial}{\partial u} G^{D}(u, t) \\
\frac{\partial^{2}}{\partial t^{2}} G^{D}(u, t)= & \mu^{2}(1-u) \frac{\partial}{\partial u}\left[(1-u) \frac{\partial}{\partial u}\right] G^{D}(u, t) \\
= & -\mu^{2}(1-u) \frac{\partial}{\partial u} G^{D}(u, t)+\mu^{2}(1-u)^{2} \frac{\partial^{2}}{\partial u^{2}} G^{D}(u, t) \\
\frac{\partial^{3}}{\partial t^{3}} G^{D}(u, t)= & \mu^{3}(1-u) \frac{\partial}{\partial u} G^{D}(u, t)-3 \mu^{3}(1-u)^{2} \frac{\partial^{2}}{\partial u^{2}} G^{D}(u, t)+ \\
& +\mu^{3}(1-u)^{3} \frac{\partial^{3}}{\partial u^{3}} G^{D}(u, t)
\end{aligned}
$$

and, analogously

$$
\begin{aligned}
\frac{\partial^{4}}{\partial t^{4}} G^{D}(u, t)= & -\mu^{4}(1-u) \frac{\partial}{\partial u} G^{D}(u, t)+7 \mu^{4}(1-u)^{2} \frac{\partial^{2}}{\partial u^{2}} G^{D}(u, t)+ \\
& -6 \mu^{4}(1-u)^{3} \frac{\partial^{3}}{\partial u^{3}} G^{D}(u, t)+\mu^{4}(1-u)^{4} \frac{\partial^{4}}{\partial u^{4}} G^{D}(u, t),
\end{aligned}
$$

we can write

$$
\frac{\partial^{j}}{\partial t^{j}} G^{D}(u, t)=\mu^{j} \sum_{l=0}^{j-1}\left\{\begin{array}{c}
j \\
j-l
\end{array}\right\}(-1)^{l}(1-u)^{j-l} \frac{\partial^{j-l}}{\partial u^{j-l}} G^{D}(u, t),
$$

where $\left\{\begin{array}{l}n \\ k\end{array}\right\}$ are the Stirling numbers of the second kind (see [14]). Thus we get (by considering that $\left.\left\{\begin{array}{l}j \\ 0\end{array}\right\}=0\right)$

$$
\begin{aligned}
\frac{\partial}{\partial t} G^{\mathcal{Y}}(u, t) & =-\lambda G^{\mathcal{Y}}(u, t)+\lambda \sum_{r=0}^{\infty} \operatorname{Pr}\left\{N_{\lambda}(t)=r\right\} \sum_{j=0}^{\infty} \frac{\mu^{j}}{j !} \sum_{l=0}^{j}\left\{\begin{array}{c}
j \\
j-l
\end{array}\right\}(-1)^{l}(1-u)^{j-l} \frac{\partial^{j-l}}{\partial u^{j-l}} G^{D}(u, r) \\
& =-\lambda G^{\mathcal{Y}}(u, t)+\lambda \sum_{r=0}^{\infty} \operatorname{Pr}\left\{N_{\lambda}(t)=r\right\} \sum_{j=0}^{\infty} \frac{\mu^{j}}{j !} \sum_{l=0}^{j}\left\{\begin{array}{l}
j \\
l
\end{array}\right\}(-1)^{j-l}(1-u)^{l} \frac{\partial^{l}}{\partial u^{l}} G^{D}(u, r) \\
& =-\lambda G^{\mathcal{Y}}(u, t)+\lambda \sum_{j=0}^{\infty} \frac{(-\mu)^{j}}{j !} B_{j}\left[(u-1) \frac{\partial}{\partial u}\right] G^{\mathcal{Y}}(u, t),
\end{aligned}
$$


where we have applied the Dobinski formula for the Bell polynomial $B_{n}$, i.e.

$$
B_{n}(x)=\sum_{k=0}^{n} x^{k}\left\{\begin{array}{l}
n \\
k
\end{array}\right\} .
$$

Equation (5.11) follows by applying the well-known exponential generating function of the Bell's polynomial (see e.g. [14), i.e.

$$
\sum_{n=0}^{\infty} \frac{t^{n} B_{n}(x)}{n !}=\exp \left\{\left(e^{t}-1\right) x\right\}
$$

Theorem 9 The probability mass function of $\mathcal{Y}(t)), t \geq 0$ satisfies the following equation

$$
\frac{d}{d t} q_{k}^{\mathcal{Y}}(t)=\lambda e^{-\mu k} \sum_{r=0}^{n_{0}-k}\left(\begin{array}{c}
r+k \\
r
\end{array}\right) q_{r+k}^{\mathcal{Y}}(t)\left(1-e^{-\mu}\right)^{r}-\lambda q_{k}^{\mathcal{Y}}(t), \quad 0 \leq k \leq n_{0},
$$

with the initial condition

$$
q_{k}^{\mathcal{y}}(0)=\left\{\begin{array}{ll}
1, & k=n_{0} \\
0, & k<n_{0}
\end{array} .\right.
$$

Proof. In order to derive the differential equation satisfied by (5.7) we rewrite the first line in (5.11) as follows:

$$
\begin{aligned}
\frac{\partial}{\partial t} G^{\mathcal{Y}}(u, t) & =\lambda \sum_{j=0}^{\infty} \frac{(1-u)^{j}\left(1-e^{-\mu}\right)^{j}}{j !} \partial_{u}^{j} G^{\mathcal{Y}}(u, t)-\lambda G^{\mathcal{Y}}(u, t) \\
& =\lambda \sum_{k=0}^{n_{0}} q_{k}^{\mathcal{Y}}(t) \sum_{j=0}^{\infty} \frac{(1-u)^{j}\left(1-e^{-\mu}\right)^{j}}{j !} \partial_{u}^{j} u^{k}-\lambda G^{\mathcal{Y}}(u, t) \\
& =\lambda \sum_{k=0}^{n_{0}} q_{k}^{\mathcal{Y}}(t) \sum_{j=0}^{\infty} \frac{\left(1-e^{-\mu}\right)^{j}}{j !} k(k-1) \ldots(k-j+1) u^{k-j} \sum_{l=0}^{j}\left(\begin{array}{l}
j \\
l
\end{array}\right)(-u)^{l}-\lambda G^{\mathcal{Y}}(u, t) \\
& =\lambda \sum_{k=0}^{n_{0}} q_{k}^{\mathcal{Y}}(t) \sum_{j=0}^{k}\left(1-e^{-\mu}\right)^{j}\left(\begin{array}{l}
k \\
j
\end{array}\right) u^{k-j} \sum_{l=0}^{j}\left(\begin{array}{l}
j \\
l
\end{array}\right)(-u)^{l}-\lambda G^{\mathcal{Y}}(u, t) \\
& =\left[l^{\prime}=k-j+l\right] \\
& =\lambda \sum_{k=0}^{n_{0}} q_{k}^{\mathcal{Y}}(t) \sum_{j=0}^{k}\left(1-e^{-\mu}\right)^{j}\left(\begin{array}{l}
k \\
j
\end{array}\right) \sum_{l^{\prime}=k-j}^{k}\left(\begin{array}{c}
j \\
k-l^{\prime}
\end{array}\right)(-1)^{j-k+l^{\prime}} u^{l^{\prime}}-\lambda G^{\mathcal{Y}}(u, t) \\
& =\lambda \sum_{k=0}^{n_{0}} q_{k}^{\mathcal{Y}}(t) \sum_{l=0}^{k} u^{l}\left(\begin{array}{c}
k \\
l
\end{array}\right) \sum_{j=k-l}^{k}\left(1-e^{-\mu}\right)^{j}\left(\begin{array}{c}
l \\
k-j
\end{array}\right)(-1)^{j-k+l}-\lambda G^{\mathcal{Y}}(u, t) \\
& =\lambda \sum_{l=0}^{n_{0}} u^{l} \sum_{k=l}^{n_{0}}\left(\begin{array}{l}
k \\
l
\end{array}\right) q_{k}^{\mathcal{Y}}(t) \sum_{j=k-l}^{k}\left(1-e^{-\mu}\right)^{j}\left(\begin{array}{c}
l \\
l-k+j
\end{array}\right)(-1)^{j-k+l}-\lambda G^{\mathcal{Y}}(u, t) \\
& =\left[j=k-l+j^{\prime}\right]
\end{aligned}
$$




$$
\begin{aligned}
& =\lambda \sum_{l=0}^{n_{0}} u^{l} \sum_{k=l}^{n_{0}}\left(\begin{array}{l}
k \\
l
\end{array}\right) q_{k}^{\mathcal{Y}}(t) \sum_{j^{\prime}=0}^{l}\left(1-e^{-\mu}\right)^{j^{\prime}+k-l}\left(\begin{array}{c}
l \\
j^{\prime}
\end{array}\right)(-1)^{j^{\prime}}-\lambda G^{\mathcal{Y}}(u, t) \\
& =\lambda \sum_{l=0}^{n_{0}} u^{l} \sum_{k=l}^{n_{0}}\left(\begin{array}{c}
k \\
l
\end{array}\right) q_{k}^{\mathcal{Y}}(t)\left(1-e^{-\mu}\right)^{k-l} e^{-\mu l}-\lambda G^{\mathcal{Y}}(u, t) \\
& =\quad[k=r+l] \\
& =\lambda \sum_{l=0}^{n_{0}} u^{l} \sum_{r=0}^{n_{0}-l}\left(\begin{array}{c}
r+l \\
l
\end{array}\right) q_{r+l}^{\mathcal{Y}}(t)\left(1-e^{-\mu}\right)^{r} e^{-\mu l}-\lambda G^{\mathcal{Y}}(u, t) .
\end{aligned}
$$

Remark 10 By comparing equation (5.12) with (5.2), we can see that, in the subordinated case the time-derivative of $q_{k}^{\mathcal{Y}}(t)$ depends on the probabilities $q_{r}^{\mathcal{Y}}(t)$, for any $k \leq r \leq n_{0}$, while, in the standard case, the derivative of $p_{k}^{D}$ depends only on $p_{k+1}^{D}$. This is due to the fact that the process $\mathcal{Y}(t), t>0$ performs downward jumps of arbitrary size, whose law can be derived from (5.7) and can be written as follows:

$$
\operatorname{Pr}\{\mathcal{Y}(t+d t)=k \mid \mathcal{Y}(t)=r+k\}=\left\{\begin{array}{c}
1-\lambda d t+\lambda d t e^{-\mu k}, \quad r=0 \\
\lambda d t\left(\begin{array}{c}
r+k \\
r
\end{array}\right)\left(1-e^{-\mu}\right)^{r} e^{-\mu k}, \quad r \geq 1 .
\end{array}\right.
$$

The first line in (5.13) means that, during the infinitesimal interval $[t, t+d t)$, either the Poisson process (the "time") does not change or it changes and all the individuals survive during a time span of unit length.

In our view the main result of this section is the density of the first passage-time through the level $k$, i.e.

$$
T_{k}^{\mathcal{Y}}=\inf \{s>0: \mathcal{Y}(s)=k\} \quad k=0, \ldots n_{0},
$$

which is presented in the following theorem.

Theorem 11 The probability density of $T_{k}^{\mathcal{Y}}$ reads, for any $k=0, \ldots, n_{0}-1$,

$$
\begin{aligned}
& \operatorname{Pr}\left\{T_{k}^{\mathcal{Y}} \in d t \mid \mathcal{Y}(0)=n_{0}\right\} / d t \\
= & \lambda e^{-\lambda t-\mu k}\left(\begin{array}{c}
n_{0} \\
k
\end{array}\right) \sum_{j=0}^{\infty} \frac{(\lambda t)^{j} e^{-\mu j k}}{j !}\left\{\left[1-e^{-\mu(j+1)}\right]^{n_{0}-k}-\left[1-e^{-\mu j}\right]^{n_{0}-k}\right\} .
\end{aligned}
$$

Proof. We start by considering that, for $k=0, \ldots, n_{0}-1$

$$
\begin{aligned}
& \operatorname{Pr}\left\{T_{k}^{\mathcal{Y}} \in d t \mid \mathcal{Y}(0)=n_{0}\right\} \\
= & \sum_{h=k+1}^{n_{0}} \operatorname{Pr}\left\{D_{\mu}\left(N_{\lambda}(t)\right)=h, D_{\mu}\left(N_{\lambda}(t+d t)\right)=k\right\} \\
= & \sum_{h=k+1}^{n_{0}} \operatorname{Pr}\left\{D_{\mu}\left(N_{\lambda}(t)\right)=h, D_{\mu}\left(N_{\lambda}(t)+d N_{\lambda}(t)\right)=k\right\} \\
= & \lambda d t \sum_{j=1}^{\infty} \sum_{h=k+1}^{n_{0}} \operatorname{Pr}\left\{D_{\mu}(j)=h, D_{\mu}(j+1)=k\right\} \operatorname{Pr}\left\{N_{\lambda}(t)=j\right\}+ \\
& +\lambda d t \operatorname{Pr}\left\{D_{\mu}(0)=n_{0}, D_{\mu}(1)=k\right\} \operatorname{Pr}\left\{N_{\lambda}(t)=0\right\}
\end{aligned}
$$




$$
\begin{aligned}
= & \lambda d t e^{-\lambda t} \sum_{j=1}^{\infty} \frac{(\lambda t)^{j}}{j !} \sum_{h=k+1}^{n_{0}}\left(\begin{array}{l}
h \\
k
\end{array}\right) e^{-\mu k}\left(1-e^{-\mu}\right)^{h-k}\left(\begin{array}{c}
n_{0} \\
h
\end{array}\right) e^{-\mu j h}\left(1-e^{-\mu j}\right)^{n_{0}-h}+ \\
& +\lambda d t e^{-\lambda t}\left(\begin{array}{c}
n_{0} \\
k
\end{array}\right) e^{-\mu k}\left(1-e^{-\mu}\right)^{n_{0}-k} \\
= & \lambda d t e^{-\lambda t-\mu k}\left(\begin{array}{c}
n_{0} \\
k
\end{array}\right) \sum_{j=1}^{\infty} \frac{(\lambda t)^{j}}{j !} \sum_{h=k+1}^{n_{0}}\left(\begin{array}{c}
n_{0}-k \\
n_{0}-h
\end{array}\right)\left(1-e^{-\mu}\right)^{h-k} e^{-\mu j h}\left(1-e^{-\mu j}\right)^{n_{0}-h}+ \\
& +\lambda d t e^{-\lambda t}\left(\begin{array}{c}
n_{0} \\
k
\end{array}\right) e^{-\mu k}\left(1-e^{-\mu}\right)^{n_{0}-k} \\
= & \lambda d t e^{-\lambda t-\mu k}\left(\begin{array}{c}
n_{0} \\
k
\end{array}\right) \sum_{j=1}^{\infty} \frac{(\lambda t)^{j}}{j !} e^{-\mu j k}\left\{\left[e^{-\mu j}\left(1-e^{-\mu}\right)+1-e^{-\mu j}\right]^{n_{0}-k}-\left[1-e^{-\mu j}\right]^{n_{0}-k}\right\}+ \\
& +\lambda d t e^{-\lambda t}\left(\begin{array}{c}
n_{0} \\
k
\end{array}\right) e^{-\mu k}\left(1-e^{-\mu}\right)^{n_{0}-k},
\end{aligned}
$$

which coincides with (5.14).

Remark 12 By integrating (5.14) we get that, for $k=0, \ldots, n_{0}-1$

$$
\operatorname{Pr}\left\{T_{k}^{\mathcal{Y}}<\infty \mid \mathcal{Y}(0)=n_{0}\right\}=e^{-\mu k}\left(\begin{array}{c}
n_{0} \\
k
\end{array}\right) \sum_{j=0}^{\infty} e^{-\mu j k}\left\{\left[1-e^{-\mu(j+1)}\right]^{n_{0}-k}-\left[1-e^{-\mu j}\right]^{n_{0}-k}\right\} .
$$

We note that the probability (5.16) has the same structure of formula (36) in [11], which is related to the iterated Poisson process, despite the fact that the outer process $D$ has decreasing paths.

Remark 13 An alternative form of (5.14), as a finite sum, can be obtained as follows

$\operatorname{Pr}\left\{T_{k}^{\mathcal{Y}} \in d t \mid \mathcal{Y}(0)=n_{0}\right\} / d t=\lambda e^{-\mu k}\left(\begin{array}{c}n_{0} \\ k\end{array}\right) \sum_{m=1}^{n_{0}-k}\left(\begin{array}{c}n_{0}-k \\ m\end{array}\right)(-1)^{m-1}\left(1-e^{-\mu m}\right) \exp \left[-\lambda t\left(1-e^{\mu(k+m)}\right)\right]$,

which, by integration, gives

$$
\operatorname{Pr}\left\{T_{k}^{\mathcal{Y}}<\infty \mid \mathcal{Y}(0)=n_{0}\right\}=e^{-\mu k}\left(\begin{array}{c}
n_{0} \\
k
\end{array}\right) \sum_{m=0}^{n_{0}-k}(-1)^{m-1}\left(\begin{array}{c}
n_{0}-k \\
m
\end{array}\right) \frac{1-e^{-\mu m}}{1-e^{-\mu(m+k)}},
$$

for any $k \geq 0$. We note that, for $k=0$, the extinction probability is given by

$$
\operatorname{Pr}\left\{T_{0}^{\mathcal{Y}}<\infty \mid \mathcal{Y}(0)=n_{0}\right\}=-\sum_{r=1}^{n_{0}}(-1)^{r}\left(\begin{array}{c}
n_{0} \\
r
\end{array}\right)=-\sum_{r=0}^{n_{0}}(-1)^{r}\left(\begin{array}{c}
n_{0} \\
r
\end{array}\right)+1=1 .
$$

For $k=n_{0}-1$, we have instead that

$$
\begin{aligned}
\operatorname{Pr}\left\{T_{n_{0}-1}^{\mathcal{Y}}<\infty \mid \mathcal{Y}(0)=n_{0}\right\} & =e^{-\mu\left(n_{0}-1\right)} n_{0} \frac{1-e^{-\mu}}{1-e^{-\mu n_{0}}} \\
& =e^{-\mu\left(n_{0}-1\right)} \frac{n_{0}}{1+e^{-\mu}+e^{-2 \mu}+\ldots+e^{-\mu\left(n_{0}-1\right)}} \\
& <e^{-\mu\left(n_{0}-1\right)} \frac{n_{0}}{n_{0} e^{-\mu\left(n_{0}-1\right)}}=1
\end{aligned}
$$

Unexpectedly enough Fig.3 (which is obtained here for $\mu=1 / 2$ ) shows that the probabilities $\operatorname{Pr}\left\{T_{k}^{\mathcal{Y}}<\infty \mid \mathcal{Y}(0)=n_{0}\right\}$ do not display a monotonic behavior for sufficiently large values of $n_{0}$. 
Figure 3: Hitting times $\operatorname{Pr}\left\{T_{k}^{\mathcal{Y}}<\infty\right\}$
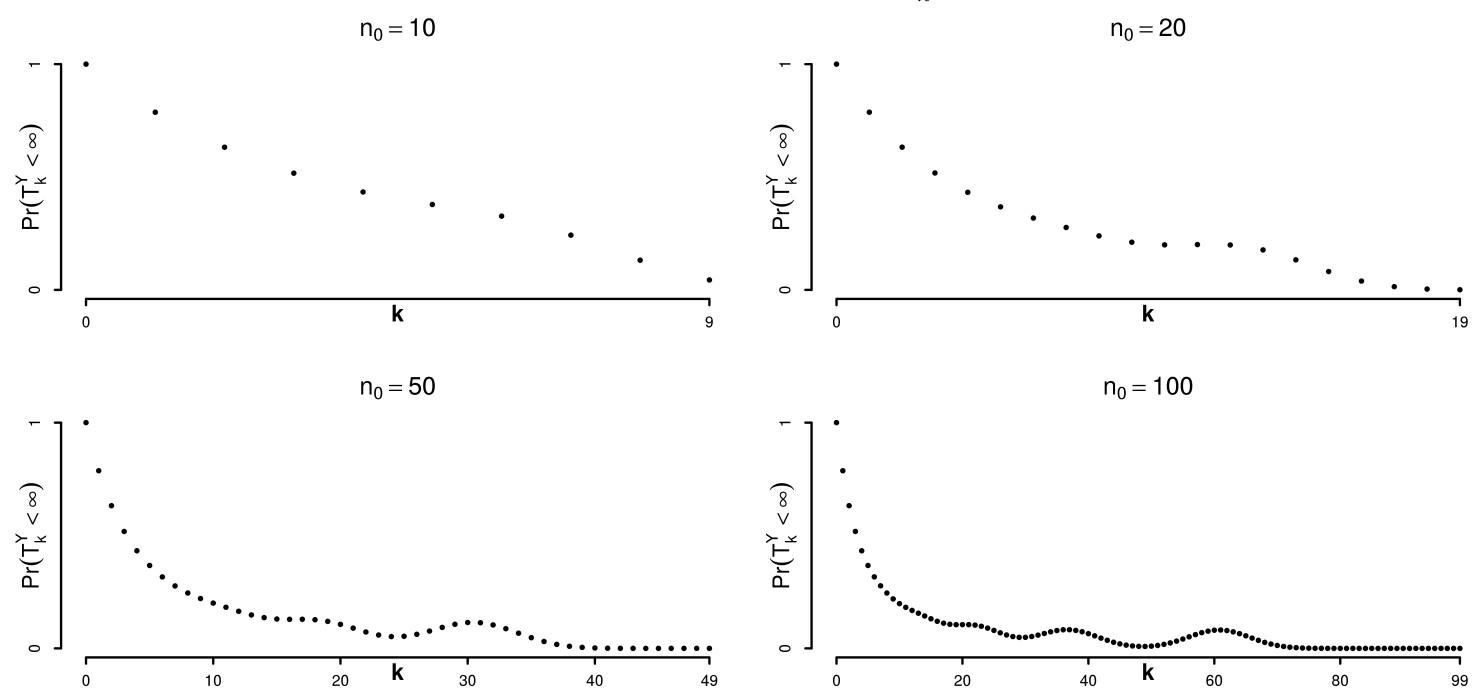

\subsection{Sublinear subordinated death process}

Let

$$
\widetilde{\mathcal{Y}}(t):=\widetilde{D}_{\mu}\left(N_{\lambda}(t)\right)
$$

where $\widetilde{D}_{\mu}$ is a sublinear death process with parameter $\mu$ and $N_{\lambda}$ is an independent Poisson process with parameter $\lambda$. Then the probability mass function reads

$$
\begin{aligned}
q_{k, n_{0}}^{\widetilde{\mathcal{Y}}_{0}}(t) & :=\operatorname{Pr}\left\{\widetilde{\mathcal{Y}}(t)=k \mid \widetilde{\mathcal{Y}}(0)=n_{0}\right\} \\
& =\sum_{l=0}^{\infty} \operatorname{Pr}\left\{\widetilde{D}_{\mu}(l)=k \mid \widetilde{D}_{\mu}(0)=n_{0}\right\} \operatorname{Pr}\left\{N_{\lambda}(t)=l\right\} \\
& =e^{-\lambda t} \sum_{j=0}^{n_{0}-k}\left(\begin{array}{c}
n_{0}-k \\
j
\end{array}\right)(-1)^{j} \exp \left\{\lambda t e^{-\mu(1+j)}\right\},
\end{aligned}
$$

for $1 \leq k \leq n_{0}$, while, for $k=0$, is given by

$$
q_{0, n_{0}}^{\widetilde{\mathcal{Y}}}(t)=e^{-\lambda t} \sum_{j=0}^{n_{0}}\left(\begin{array}{c}
n_{0} \\
j
\end{array}\right)(-1)^{j} \exp \left\{\lambda t e^{-\mu j}\right\} .
$$

Clearly $q_{0, n_{0}}^{\widetilde{\mathcal{Y}}}$ is a decreasing function of the initial number of individuals, as can be directly checked, by considering (5.3). The expected value of $\widetilde{\mathcal{Y}}(t), t \geq 0$ can be evaluated, by considering (5.4), as follows

$$
\begin{aligned}
\mathbb{E} \widetilde{\mathcal{Y}}(t) & =e^{-\lambda t} \sum_{j=0}^{\infty}\left\{n_{0}+1-e^{\mu j}\left[1-\left(1-e^{-\mu j}\right)^{n_{0}+1}\right]\right\} \frac{(\lambda t)^{j}}{j !} \\
& =n_{0}+1-e^{-\lambda t\left(1-e^{\mu}\right)}+\sum_{j=0}^{\infty}\left(1-e^{-\mu j}\right)^{n_{0}+1} \frac{\left(\lambda t e^{\mu}\right)^{j}}{j !} \\
& \leq n_{0}-\left(1-e^{-\lambda t\left(1-e^{-\mu}\right)}\right)
\end{aligned}
$$


In the case of the subordinated sublinear death process, we have no markovianity and thus we cannot evaluate explicitly the distribution of the hitting times $T_{k}^{\widetilde{\mathcal{Y}}}, 0 \leq k \leq n_{0}-1$. For this reason we consider here the instant of the first downcrossing of the level $k$, i.e.

$$
V_{k}^{\tilde{\mathcal{Y}}}=\inf \{s>0: \widetilde{\mathcal{Y}}(s) \leq k\}, \quad 0 \leq k \leq n_{0}-1 .
$$

For $k \geq 1$, we can write

$$
\begin{aligned}
\operatorname{Pr}\left\{V_{k}^{\tilde{\mathcal{Y}}}>t\right\} & =\operatorname{Pr}\{\tilde{\mathcal{Y}}(t) \geq k\} \\
& =[\text { by } 5.17]] \\
& =\sum_{l=k}^{n_{0}} \sum_{r=0}^{n_{0}-l}\left(\begin{array}{c}
n_{0}-l \\
r
\end{array}\right)(-1)^{r} \exp \left\{-\lambda t\left(1-e^{-\mu(1+r)}\right)\right\} \\
& =\sum_{r=0}^{n_{0}-k}(-1)^{r} \exp \left\{-\lambda t\left(1-e^{-\mu(1+r)}\right)\right\} \sum_{l=k}^{n_{0}-r}\left(\begin{array}{c}
n_{0}-l \\
r
\end{array}\right) .
\end{aligned}
$$

The inner sum can be treated as follows

$$
\begin{aligned}
\sum_{l=k}^{n_{0}-r}\left(\begin{array}{c}
n_{0}-l \\
r
\end{array}\right) & =\sum_{l=0}^{n_{0}-r-k}\left(\begin{array}{c}
n_{0}-l-k \\
r
\end{array}\right) \\
& =[\text { by eq. }(2.25) \text { in [2] }] \\
& =\left(\begin{array}{c}
n_{0}-k+1 \\
r+1
\end{array}\right),
\end{aligned}
$$

so that we get

$$
\operatorname{Pr}\left\{V_{k}^{\widetilde{\mathcal{Y}}}>t\right\}=\sum_{r=1}^{n_{0}-k+1}(-1)^{r-1}\left(\begin{array}{c}
n_{0}-k+1 \\
r
\end{array}\right) \exp \left\{-\lambda t\left(1-e^{-\mu r}\right)\right\},
$$

which vanishes, for $t \rightarrow \infty$, for any $k \geq 1$. For $k=0$, by applying (5.18) we have, instead, the following extinction probability:

$$
\operatorname{Pr}\left\{V_{0}^{\tilde{\mathcal{Y}}}<t\right\}=\operatorname{Pr}\{\widetilde{\mathcal{Y}}(t)=0\}=e^{-\lambda t} \sum_{j=0}^{n_{0}}\left(\begin{array}{c}
n_{0} \\
j
\end{array}\right)(-1)^{j} \exp \left\{\lambda t e^{-\mu j}\right\},
$$

which, for $t \rightarrow \infty$, is equal to 1 .

\section{Acknowledgement}

We thank Dr. Bruno Toaldo for providing the figures presented in this paper.

\section{References}

[1] Alipour M., Beghin L., Rostamy D. (2013), Generalized fractional non-linear birth processes, Method. Comput. Applied Probab., 1-16.

[2] Beghin L., Orsingher E. (2012), Poisson process with different Brownian clocks, Stochastics, 84 (1), 79-112. 
[3] Cahoy D., Sibatov R., Uchaikin V.(2008), Fractional processes: from Poisson to branching one. International Journal of Bifurcation and Chaos (in Applied Sciences and Engineering), 18(9), 2717-2725.

[4] Cahoy D., Polito F. (2012), Simulation and estimation for the fractional Yule process, Method. Comput. Applied Probab., 14 (2), 383-403.

[5] Cahoy D., Polito F. (2014), Parameter estimation for fractional birth and fractional death processes. Statistics and Computing, 24(2), 211-222.

[6] Di Crescenzo A., Martinucci B., Zacks, S. (2015), Compound Poisson process with Poisson subordinator. J. Appl. Probab., 52 (2), 1-17.

[7] Ding X., Giesecke K., Tomecek P.I. (2009), Time-changed birth processes and multi-name credit derivatives, Operations Research, 57 (4), 990-1005.

[8] Ding X. (2010), Time-changed birth processes, random thinning, and correlated default risk, Stanford Univ., PhD thesis.

[9] Donnelly, P., Kurtz, T., Marjoram, P. (1993), Correlation and variability in birth processes. J. Appl. Probab. 30(2), 275-284.

[10] Kumar A., Nane E., Vellaisamy P. (2011), Time-changed Poisson processes, Stat. Probab. Letters 81 (12), 1899-1910.

[11] Orsingher E., Polito F. (2012), Compositions, random sums and continued random fractions of Poisson and fractional Poisson processes. Journal of Statistical Physics, 148, (2), 233-249.

[12] Orsingher E., Polito F., Sakhno L. (2010), Fractional non-linear, linear and sublinear death processes. Journal of Statistical Physics, 141, (1), 68-93.

[13] Orsingher E., Ricciuti C., Toaldo B. (2014), Population models at stochastic times. ArXiv 1407.1173.

[14] Riordan J. (1958), An Introduction to Combinatorial Analysis, John Wiley and Sons. 